\title{
BMJ Open Relationship between triglyceride glucose index and the incidence of non- alcoholic fatty liver disease in the elderly: a retrospective cohort study in China
}

Chen Huanan, ${ }^{1}$ Li Sangsang, ${ }^{2}$ Adwoa Nyantakyiwaa Amoah, ${ }^{1}$ Bo Yacong, ${ }^{3}$ Chen Xuejiao (D) , ${ }^{4}$ Shi Zhan, ${ }^{5}$ Wan Guodong, ${ }^{6}$ Huang Jian, ${ }^{7}$ Shi Songhe (D) , 8 Lyu Quanjun ${ }^{1,9}$

To cite: Huanan C, Sangsang L, Amoah AN, et al. Relationship between triglyceride glucose index and the incidence of non-alcoholic fatty liver disease in the elderly: a retrospective cohort study in China. BMJ Open 2020;10:e039804. doi:10.1136/ bmjopen-2020-039804

- Prepublication history and additional material for this paper is available online. To view these files, please visit the journal online (http://dx.doi.org/10. 1136/bmjopen-2020-039804).

Received 14 May 2020 Revised 15 August 2020 Accepted 18 August 2020
D) Check for updates

(c) Author(s) (or their employer(s)) 2020. Re-use permitted under CC BY-NC. No commercial re-use. See rights and permissions. Published by BMJ.

For numbered affiliations see end of article.

Correspondence to Dr Lyu Quanjun; lqjnutr@zzu.edu.cn and Professor Shi Songhe; zzussh@126.com

\section{ABSTRACT}

Objective Non-alcoholic fatty liver disease (NAFLD) is one of the major causes of liver-related diseases but relationship between triglyceride glucose (TyG) and NAFLD in the elderly is not reported yet. In this study, we investigated the role of TyG index for predicting the incidence of NAFLD in the elderly.

Design and setting This is a prospective cohort study in Henan, China, from 2011 to 2018.

Participants and methods In total, 46693 elderly who participated in a routine physical examination programme from 2011 to 2018 were included in this study. TyG index was calculated as In (fasting triglyceride $(\mathrm{mg} / \mathrm{dL}) \times$ fasting plasma glucose $(\mathrm{mg} / \mathrm{dL}) / 2$ ), while NAFLD was defined as hepatic steatosis after excluding other causes based on the results of abdominal ultrasonography; Cox regression model was performed to explore the relationship between TyG index and NAFLD. Also, mediation effect was used to analyse the role of the TyG index in WHtR (waist-to-height ratio) and NAFLD.

Results During the 149041 person-years follow-up, a total of 5660 NAFLD events occurred (3.80/100 personyears). After adjusting for potential confounding factors, quartiles 4 of TyG index significantly increased the incidence of NAFLD compared with quartile 1, the HRs and 95\% Cl were 1.314 (1.234 to 1.457). In addition, TyG index played a partial mediating role in the relationship between WHtR and NAFLD and indirect effect was 1.009 (1.006 to 1.011).

Conclusion Higher TyG index was associated with higher risk of NAFLD in the aged, and therefore, TyG index may be a novel predictor for incidence of NAFLD. Further, regular examination and evaluation of the TyG index might be useful for controlling the occurrence of NAFLD.

\section{INTRODUCTION}

Non-alcoholic fatty liver disease (NAFLD) encompasses a series of spectrum of liver diseases, ranging from simple steatosis, nonalcoholic steatohepatitis, cirrhosis to hepatocellular carcinoma. ${ }^{1}$ With the change of
Strengths and limitations of this study

- Data for this cohort study were retrieved from a large regular physical examination in Henan, China.

- Restricted cubic spline analysis and mediation effect were used in this study, which can reflect the relationship between triglyceride glucose index and non-alcoholic fatty liver disease more realistically.

- The sample size and statistical power were sufficient in this study.

- The severity of NAFLD in this cohort study was not available when data were retrieved.

- Some participants were excluded in this study because they did not have abdominal ultrasound testing.

lifestyle and increase of obesity, NAFLD currently exceeds viral hepatitis and is becoming the most common chronic liver disease, affecting about one-quarter of the common population in the world. ${ }^{23}$ NAFLD is one of the major causes of liver-related disease such as cirrhosis, hepatocellular carcinoma and liver transplantation. Moreover, NAFLD is also associated with higher prevalence and incidence of cardiovascular disease such as coronary, cerebrovascular and peripheral vascular disease. ${ }^{45}$ In the next decade, NAFLD is expected to become the leading cause for liver transplantation in the USA instead of hepatitis C. ${ }^{6}$

The development of NAFLD is a complex process involving genetic and environmental factors. As an organ of metabolism, the disorder of glucose and lipid metabolism plays an important role in the progress of NAFLD. ${ }^{7}$ Several studies found that insulin resistance (IR) could increase the risk of NAFLD even without the existence of type 
2 diabetes mellitus and IR may be the genesis of steatosis. ${ }^{7-10}$ Furthermore, as an early marker of IR, triglyceride glucose (TyG) has been proposed to be an inexpensive and reliable surrogate to $\mathrm{IR}^{11}{ }^{12}$ Yet, research about the relationship between TyG index and the risk of NAFLD are limited. ${ }^{913}$ Additionally, as far as we know, the association between TyG index and the incidence of NAFLD in the elderly has not been reported.

WHtR (waist-to-height ratio) is a maker of abdominal obesity and studies have also reported that WHtR is related to $\mathrm{IR}^{1415}$ and also the higher risk of NAFLD. ${ }^{16-22}$ Whether there is an existing effect of TyG index on the relationship between WHtR and NAFLD and how it affects NAFLD is still unclear. This retrospective cohort study therefore sought to explore the relationship between baseline level of TyG index and the incidence of NAFLD.

\section{PARTICIPANTS AND METHODS \\ Participants}

We retrospectively analysed the 99997 participants who had the data of liver ultrasonography, fasting triglyceride (TG) as well as fasting plasma glucose (FPG) in physical examination programme in Xinzheng, Henan Province, in Central China City from January 2011 to December 2018. This physical examination programme was for the local residents over 60 years old and was supported by the government of Xinzheng, Henan Province in China. Individuals with any of the following criteria were excluded: (1) missing follow-up; (2) participants with NAFLD at baseline; (3) participants with hepatitis $\mathrm{B}$ or $\mathrm{C}$ virus or having a history of excess alcohol intake (the threshold for women was $<20 \mathrm{~g} /$ day and for men $<30 \mathrm{~g} /$ day). A total of 46693 eligible participants were included in this cohort study (figure 1). The data sets generated and/or analysed during the current study are available on request. Given the retrospective nature of the research, the requirement for informed consent was waived. Patients were not involved in the recruitment and conduct of the study.

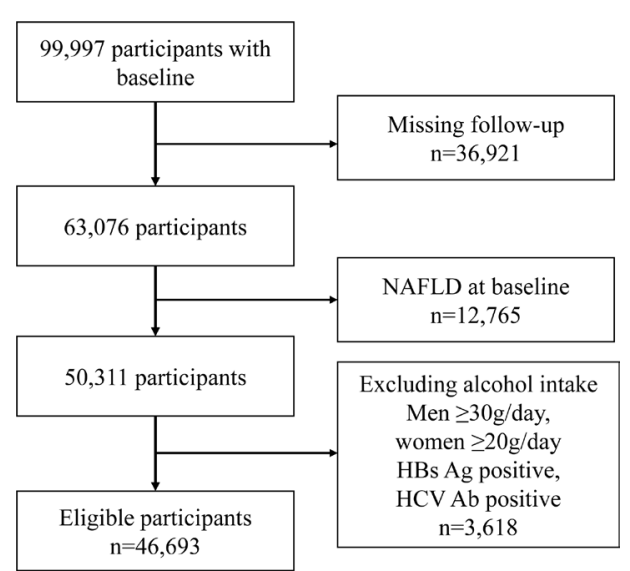

Figure 1 Flow chart for participants exclusions performed. HBs Ag, hepatitis B surface antigen; HCV Ab, hepatitis C virus antibody; NAFLD, non-alcoholic fatty liver disease.

\section{Data collection}

Demographic data and clinical information of the participants were collected when they underwent health check-up. Demographic data included age, sex, excess alcohol intake (yes/no), current smoking status (yes/no), lives alone (yes/no) and exercises regularly (yes/no). The definition of current smoking was that participants used to smoke 100 cigarettes or above in their lifetime, and now are still smoking regularly. Excessive drinking was defined as drinking more than $30 \mathrm{~g}$ /day for men and $20 \mathrm{~g} /$ day for women. Exercising regularly was defined as more than three times a week, and 30 min of moderateintensity exercise each time. The clinic records included anthropometric measurements (such as height, weight, blood pressure and waist circumference) and laboratory data. Height measurement required the participants stand straight on the ground without shoes, with their hips and heels against the wall; to measure the weight, the participants removed shoes and wore light clothing. Blood pressure of the participants, in the sitting position after 5 min of rest, was measured twice using an electronic sphygmomanometer (Omron HEM-7125, Kyoto, Japan), and the mean value of the two measurements was recorded. Waist circumference was measured at the midpoint of the distance between the lowest costal ridge and the upper border of the iliac crest. After fasting for 8 hours, the blood samples of participants were collected to determine the level of FPG, total cholesterol (TC) and TG using a biochemical detector (DIRUI CS380, Changchun, China). Alanine aminotransferase (ALT), aspartate transaminase (AST) and total bilirubin (TB) were detected by DIRUI CS380. The TyG index were calculated as the ln (fasting TG $(\mathrm{mg} / \mathrm{dL}) \times \mathrm{FPG}(\mathrm{mg} / \mathrm{dL}) / 2)$. WHtR was defined as waist circumference $(\mathrm{cm}) /$ height $(\mathrm{cm})$.

\section{NAFLD definition}

All participants of this study underwent liver ultrasonography (SIUI CZXL-38G, Shantou, China). The results of ultrasound prompted the existence of steatohepatitis: enhanced liver echogenicity, echogenicity greater in liver than kidneys, deep attenuation and vascular blurring, ${ }^{23}$ and after excluding the steatohepatitis caused by alcohol, viruses and drugs, was defined as NAFLD. All ultrasound examinations were performed by an experienced professional radiologist.

\section{Statistics}

Categorical variables were showed as proportions while continuous variables were presented as means $\pm \mathrm{SD}$ or median $\pm \mathrm{IQR}$. ANOVA/two-paired sample t-test (continuous variables, normal distribution) and $\chi^{2}$ test (categorical variables) were used to compare the difference in different groups. Logistic regression for categorical variables and linear regression for continuous variables were used to obtain the $\mathrm{p}$ value for trend.

Cox regression models were used to explore the relationship between TyG index and the incidence of NAFLD, the lowest quartiles of TyG index was defined 
Table 1 Baseline characteristics of included participants according to the follow-up outcome

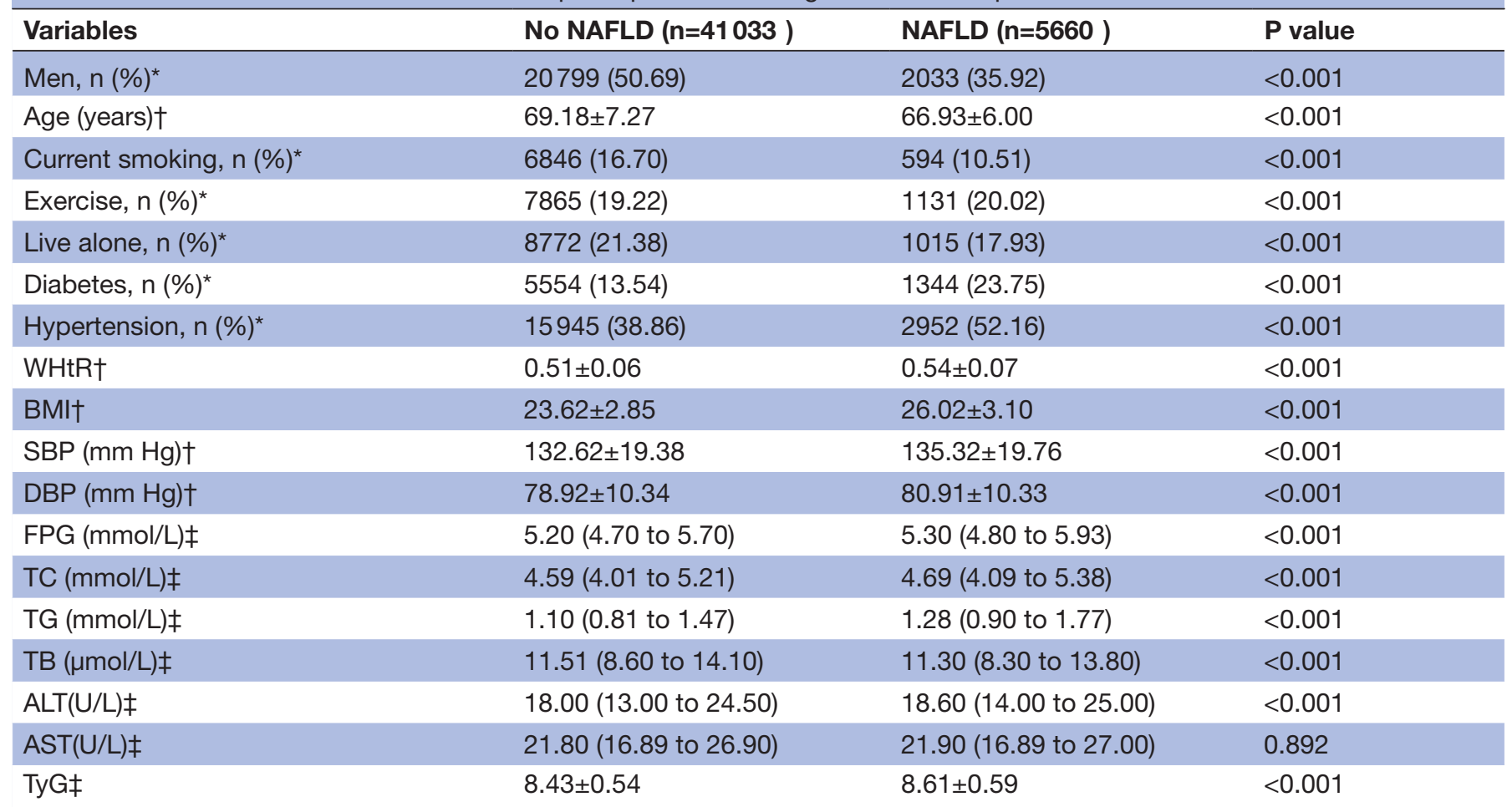

*n (\%).

†Mean (SD).

¥Median (IQR).

ALT, alanine aminotransferase; AST, aspartate transaminase ; BMI, body mass index; DBP, diastolic blood pressure; FPG, fasting plasma glucose; NAFLD, non-alcoholic fatty liver disease ; SBP, systolic blood pressure; TB, total bilirubin; TC, total cholesterol; TG, triglyceride; TyG, triglyceride glucose; WHtR, waist-to-height ratio.

as the reference. HRs and CI of NAFLD in quartiles and continuous were expressed in separate models. To assess the relationship across increasing quartiles, $p$ value for trend tests were used by entering median value in each quartile in Cox regression models.

Area under the receiver operating characteristic curves (AUCs) were used to evaluate the ability of the baseline TyG index to predict the risk of NAFLD. Restricted cubic spline models were used to explore whether there was a non-linear relationship between continuous and occurrence of NAFLD. ${ }^{24}$ Mediation analysis used a Cox regression to study the mediate effect of TyG index between WHtR and NAFLD.

Mediation analysis was conducted using R V.3.5.0 ( R Foundation for Statistical Computing, Vienna, Austria) and Restricted cubic spline were performed in Stata V.12.0 (Stata Corp, College Station, Texas, USA). The other analyses were performed by SPSS software, V.21.0 (SPSS, Chicago, Illinois, USA). Probability values for statistical tests, where two-tailed $\mathrm{P}$ values $<0.05$ were regarded as significant.

\section{RESULT}

Baseline characteristics of included participants

A total of 46693 participants were included in this study. The baseline characteristics of included participants based on follow-up are presented in table 1 . The mean age of participants was 68.91 (7.17) and $48.89 \%$ were men. The average follow-up was 3.19 (1.52) years, during the 149041 person-years follow-up, 5660 participants developed NAFLD (3.80/100 person-years). Participants who developed NAFLD during follow up had higher level of body mass index (BMI), WHtR, TC, TG, FPG, TyG index, diastolic blood pressure, systolic blood pressure, ALT and TB compared with no-NAFLD. And the incidence of NAFLD was higher in younger, current smoking, regular exercise, with diabetes and hypertension but the baseline level of AST had no association with the incidence of NAFLD.

\section{Association between TyG index and NAFLD}

The relationship between TyG index and the incidence of NAFLD are shown in table 2. Compared with quartile 1 of TyG index, the HR and its corresponding $95 \%$ CI for quartile 2, quartile 3 and quartile 4 were 1.056 (0.971 to 1.149 ), 1.300 (1.200 to 1.408 ) and 1.972 (1.832 to 2.123), respectively. Risk for NAFLD was significantly higher with increasing quartiles of TyG for the $p$ value of trend of linearity $<0.001$. In addition, even after adjusting for possible confounding factors, the risk of quartile 3 of TyG index (1.314 (1.234 to 1.457)) on the incidence of NAFLD still existed. In order to further verify the relationship between the TyG index and the incidence of 
Table 2 Relationship between TyG index and the risk of NAFLD

\begin{tabular}{lllll} 
& & & & \\
\cline { 2 - 5 } & NyG index & Unadjusted & Model 1 & Model 2 \\
\hline As continuous & 50037 & $1.697(1.619$ to1.779) & $1.577(1.504$ to 1.653) & $1.265(1.200$ to 1.334) \\
Quartile 1 & 12556 & Reference & Reference & Reference \\
Quartile 2 & 12458 & $1.056(0.971$ to 1.149$)$ & $1.035(0.951$ to 1.126) & $0.980(0.895$ to 1.073) \\
Quartile 3 & 12522 & $1.300(1.200$ to 1.408) & $1.259(1.162$ to 1.364) & $1.090(0.999$ to 1.190) \\
Quartile 4 & 12501 & $1.972(1.832$ to 2.123) & $1.783(1.655$ to 1.920) & $1.314(1.234$ to 1.457) \\
\hline P for trend & & $<0.001$ & $<0.001$ & $<0.001$ \\
\hline
\end{tabular}

Quartile $1 \leq 8.11 ; 8.12<$ Quartile $2 \leq 8.44 ; 8.45<$ Quartile $3 \leq 8.78 ; 8.79<$ Quartile 4 .

Model 1: Adjusted age and sex.

Model 2: Model 1 plus living alone, current smoking, exercise, waist-to-height ratio, systolic blood pressure, diastolic blood pressure, alanine aminotransferase, aspartate aminotransferase, total bilirubin, total cholesterol and diabetes.

NAFLD, non-alcoholic fatty liver disease; TyG, triglyceride glucose.

NAFLD, TyG index was used as continuous various for the above analysis and the results also proved that a higher level of TyG increased the risk of NAFLD (1.265 (1.200 to 1.334)), even after adjusting for possible confounding factors. These results proved that the baseline level of TyG was associated with the risk of NAFLD, thus the higher the TyG level, the higher incidence of NAFLD.

Since the majority of the participants could not respond on their history of lipid-lowering therapy or antidiabetic drugs, we failed to access information about therapy. Sensitivity analysis $(\mathrm{n}=37428)$ was executed after excluding participants with diabetes, cardiovascular disease and stroke, who might take medicine that affects level of FPG and TG. The results of sensitivity analysis (online supplemental table 1) also suggested that higher level of TyG significantly increased the incidence of NAFLD, which were similar to the findings in total population.

\section{The ability of TyG to predict NAFLD}

The ROC curves were used to evaluate the ability of baseline level of TyG to predict the development of NAFLD. The best cut-off value for TyG index to diagnose NAFLD was 8.63, and its corresponding area under the ROCs (AUCs) was 0.60 (95\%CI: 0.58 to 0.61 ), shown in figure 2A. Subgroup analysis was performed to further analyse the influence of sex on the ability of TyG index to predict the incidence of NAFLD, the best cut-off value (8.68) was lower in men than women (8.75), and corresponding AUCs was 0.587 (95\% CI: 0.573 to 0.600 )

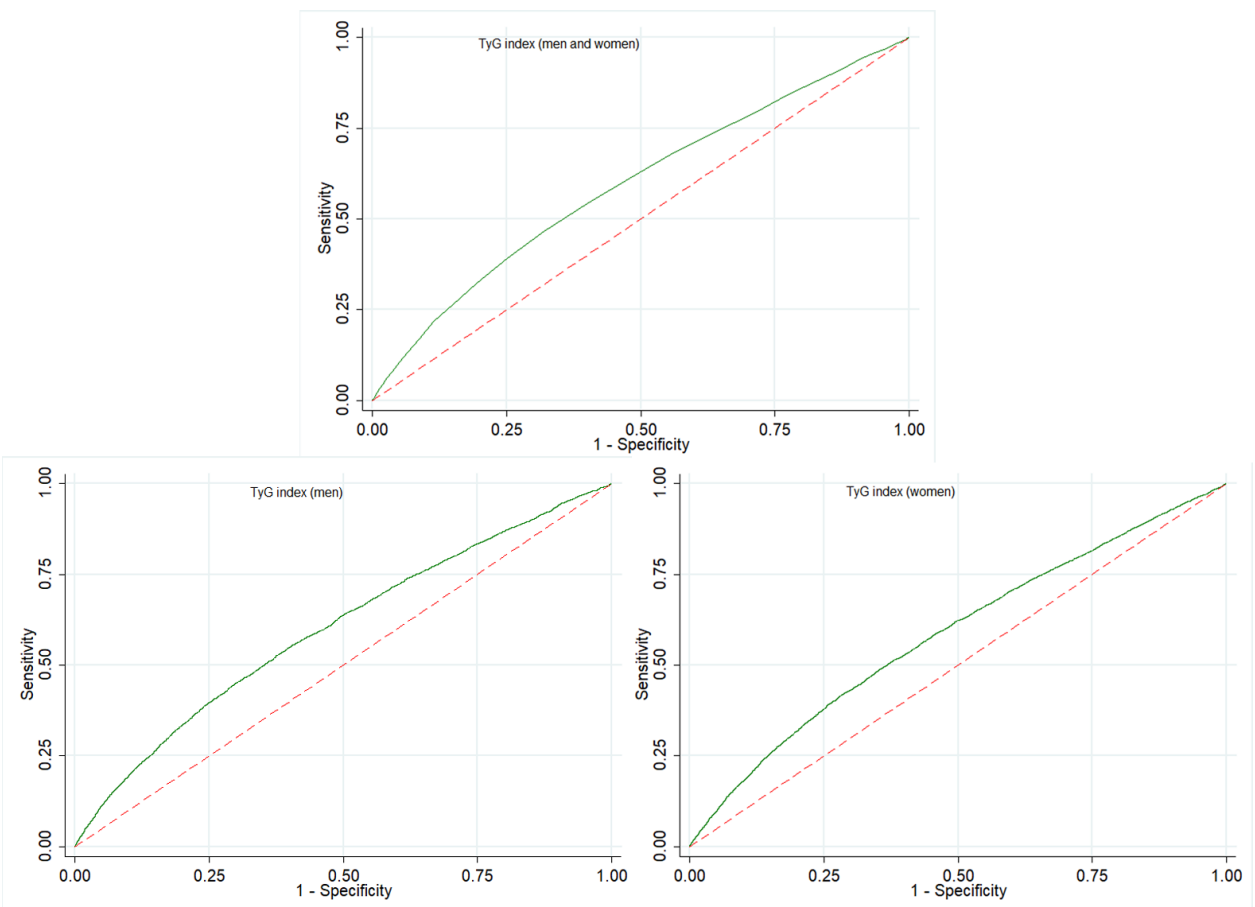

Figure 2 ROC curves of baseline TyG to predict the incidence of NAFLD among those aged 60 years or older. NAFLD, nonalcoholic fatty liver disease; ROC, receiver operating characteristic; TyG, triglyceride glucose. 

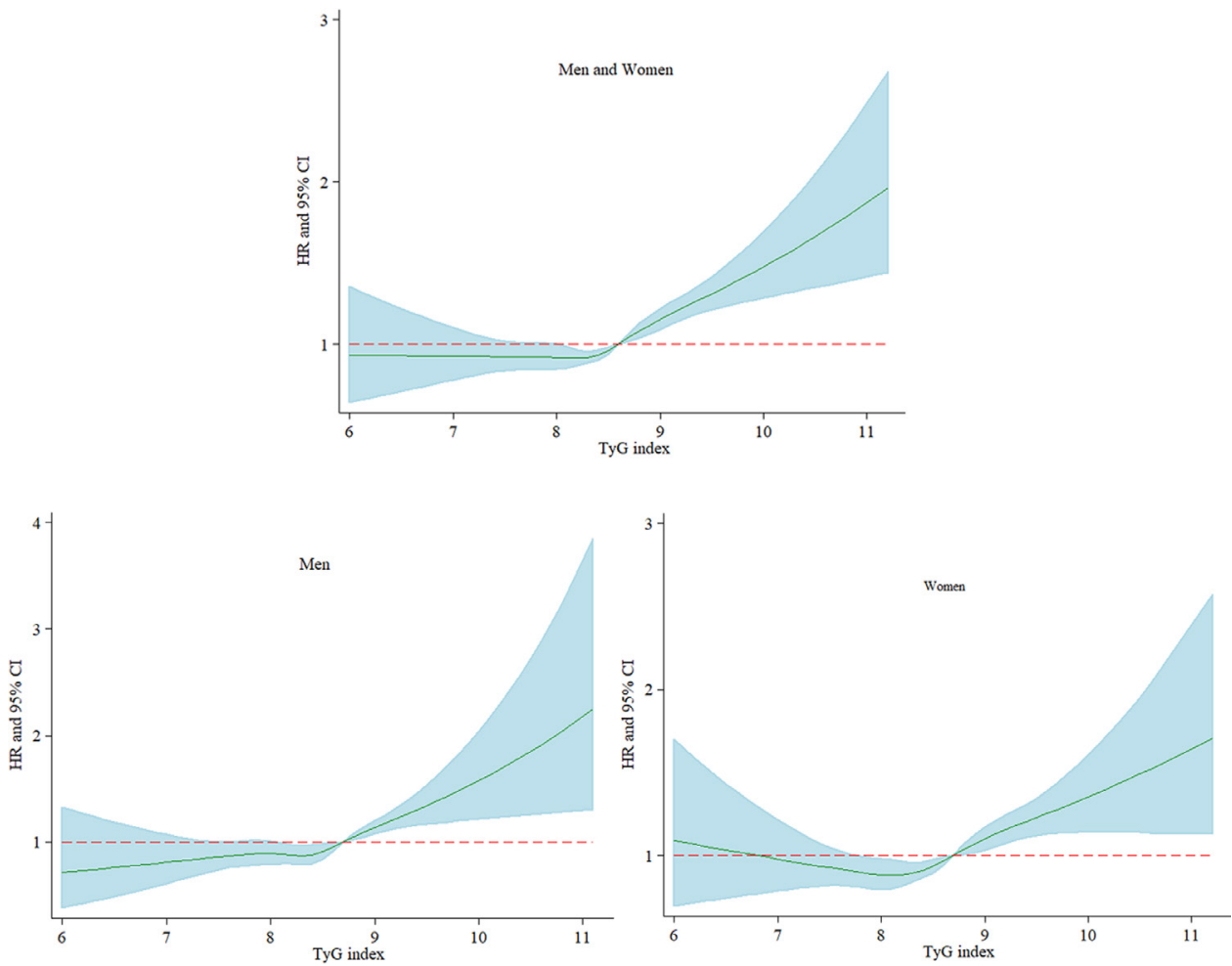

Figure 3 Restricted cubic spline graph of risk of TyG index and the development of NAFLD. Multivariate adjusted HRs of NAFLD increased during follow-up when the baseline level of TyG index was 8.63 and above. Adjusted variables including age, sex (not for sex subgroup analysis), living alone, current smoking, exercise, waist-to-height ratio, systolic blood pressure, diastolic blood pressure, alanine aminotransferase, aspartate aminotransferase, total bilirubin and total cholesterol. TyG, triglyceride glucose; NAFLD, non-alcoholic fatty liver disease.

for men and 0.584 (95\% CI: 0.573 to 0.594 ) for women (figure 2B,C).

\section{Restricted cubic spline analysis to evaluate the relationship between TyG and NAFLD}

In order to further explore the relationship between TyG and the risk of NAFLD, restricted cubic spline graph was used to analyse the dose-response relationship between TyG and the incidence of NAFLD. As shown in figure 3A, there was a non-linear relationship between level of TyG index and the risk of NAFLD based on the adjusted Cox regression model. This result proved again that higher level of TyG was associated with higher incidence of NAFLD. Subgroup analyses also found that there was a non-linear relationship between TyG index and incident NAFLD both in men and women (figure 3B,C).

\section{Mediation effects}

In previous analysis, we found that WHtR was associated with the incidence of NAFLD; what's more, WHtR was also associated with TyG index ( $\mathrm{p}<0.05$, and the data isn't shown). If WHtR would lead to IR and TyG index was a reliable and cheap surrogate indicator for IR, then there might be a mediating effect of TyG index between WHtR and NAFLD. Therefore, mediation analysis was used to confirm whether there was a mediating effect and to what extent TyG index affects the relationship between WHtR and NAFLD. Results (table 3 and figure 4) of mediation analysis revealed that the total effect of WHtR on NAFLD was 1.476 (1.437 to 1.517$)$ and the direct effect was 1.463 (1.423 to 1.503 ). Therefore, TyG index played a partial role and the indirect effect was 1.009 (1.006 to 1.011). This result may indicate that abdominal obesity may lead to disorders of glycolipid, dyslipidaemia, which can lead to an increase of the TyG index.

The effect of WHtR on NAFLD was mediated by baseline level TyG index by adjusted potential confounders namely age, sex, living alone, current smoking, exercise, systolic blood pressure, diastolic blood pressure, ALT, AST, TB and TC.

\section{DISCUSSION}

With the acceleration of the ageing process, the health of the elderly has gradually become an important issue in the society. NAFLD, a common liver disease in China, has

Table 3 Mediation analysis of the relationship between TyG index and NAFLD by WHtR

\begin{tabular}{ll}
\hline Effect & HR (95\% Cl) \\
\hline Total effect & $1.476(1.437$ to 1.517$)$ \\
Direct effect & $1.463(1.423$ to 1.503$)$ \\
Indirect effect & $1.009(1.006$ to 1.011$)$ \\
\hline
\end{tabular}

NAFLD, non-alcoholic fatty liver disease; TyG, triglyceride glucose; WHtR, waist-to-height ratio. 


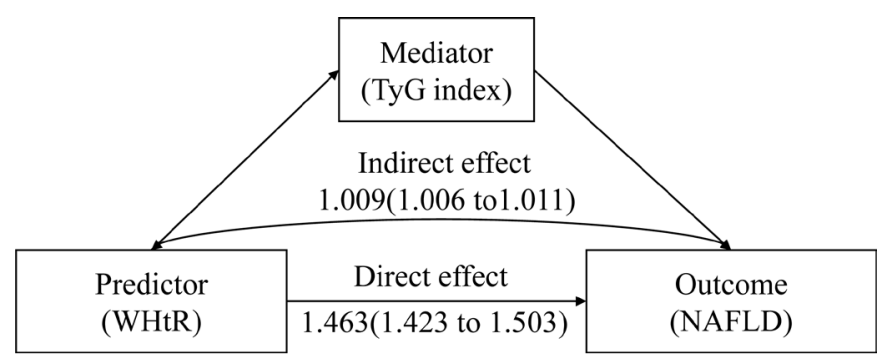

\begin{tabular}{|c|c|c|}
\hline $\begin{array}{c}\text { Predictor } \\
\text { (WHtR) }\end{array}$ & \begin{tabular}{c} 
Total effect \\
\cline { 3 - 3 }
\end{tabular} & $\begin{array}{c}\text { Outcome } \\
\text { (NAFLD) }\end{array}$ \\
\hline
\end{tabular}

Figure 4 The mediate effect of TyG index on WHtR and NAFLD. NAFLD, non-alcoholic fatty liver disease; TyG, triglyceride glucose; WHtR, waist-to-height ratio.

become one of the main health problems of the elderly. Although there were some studies about TyG index and NAFLD, research about TyG index and the incidence of NAFLD in older adults has not been reported.

In this retrospective cohort study, we found the incidence of NAFLD was 3.80/100 person-years among the elderly over 60 years, which was lower than a cohort study of Korea (the incidence of NAFLD was 4.84/100 person-years) ${ }^{25}$ Probably, the age of the participants in the Korean cohort study (mean age was 36.1) was younger than ours and age could affect the prevalence and severity of NAFLD. ${ }^{26}$ Additionally, the incidence of NAFLD decreased as the age increased in this study, $p_{\text {value }}$ for trend $<0.001$ (data not presented in this research). A retrospective cohort study on TyG index and NAFLD in Japan also found that higher TyG was associated with higher risk of NAFLD, which was consistent with our results. ${ }^{13}$ As far as we know, this research is the first study to focus on the effect of TyG index on the incidence of NAFLD in the elderly in China. Our study also reported the relationship between TyG index was non-linear hence the incidence of NAFLD would increase significantly when TyG is above 8.63. Furthermore, the results of the mediation effect suggested that TyG index was not an independent factor for the development of NAFLD, but a partial mediator between WHtR and NAFLD.

The results of ROC suggested that 8.63 was the best cut-off value of TyG index for predicting the incidence of NAFLD. The cut off value of TyG index in our study was higher than Simental-Mendía et al's, ${ }^{27}$ which might be due to the following reasons. First, the participants were different between the two studies, in Simental-Mendía et al's $^{27}$ research, the participants were asymptomatic women aged 20 to 65 years in Mexico, while in our study, the participants were 60 years or older and from China (including men and women); moreover, the cut-off value in our research was similar to several studies from Asia; ${ }^{1328}{ }^{29}$ Second, study design of Simental-Mendía et $a l^{27}$ is different from ours; what's more, the methods to diagnose NAFLD were different; additionally, the mean values of TyG index were different. The above might be the reasons that resulted in different cut-off value. TyG index is an inexpensive and reliable surrogate index for IR and plays an important role in the development and progression of NAFLD. Consequently, even in the absence of diabetes, IR can lead to changes from normal liver to NAFL to nonalcoholic steatohepatitis (NASH). ${ }^{10}$ Various studies have confirmed that IR is closely related to the occurrence and development of NAFLD. ${ }^{30-33}$

The mechanism of IR on NAFLD could be explained by the following reasons. On one hand, IR has a direct effect on metabolism of glucose and lipid, and thus participates in the incidence and development of NAFLD. ${ }^{30}$ Insulin resistance reduces glucose uptake in the adipose tissues and muscles, and reduces the hydrolysis of TGs in adipose tissue. Meanwhile, high insulin levels can increase the uptake of free fatty acids in the liver and the synthesis of TG, causing excessive accumulation of fat in the liver, which could initiate steatosis and then lead to the occurrence of NAFLD. ${ }^{7345}$ On the other hand, IR is always linked to chronic mild inflammation caused by the release of inflammatory factors, such as tumour necrosis factor- $\alpha$, interleukin (IL)-6, IL-1 and monocyte chemotactic protein-1, which can, in turn, promote IR and participate in the development and progression of NAFLD. ${ }^{36} 37$

Obesity is a key factor in the development and progression of NAFLD. It has been documented that BMI is associated with incidence of NAFLD. ${ }^{38}{ }^{39}$ While growing evidence suggested that the determinant of IR is not the degree of obesity, but the distribution of fat. ${ }^{40-43}$ Central obesity can lead to inflammatory, oxidative stress and metabolic disorders, which are related to the development of IR. ${ }^{44}$ In this study, WHtR, an indicator of central obesity, was associated with NAFLD and the effect was mediated by TyG index among the study participants. It could be that central obesity leads to an increase in the TyG index and thus results in NAFLD.The major strength of this study was a large sample size retrospective cohort study which included 46693 participants aged more than 60 years. It is believed a cohort study could better reflect the real relationship between TyG index and NAFLD. Moreover, we established a non-linear relationship between TyG index and NAFLD using cubic spline graph analysis. Furthermore, WHtR could influence the incident of NAFLD partial mediated by TyG index, that is, TyG index played a mediation effect between WHtR and NAFLD in this study. However, there were several limitations in this study. First, liver biopsy is gold standard to diagnose NAFLD, while, in this study, we executed abdominal ultrasound to diagnosis NAFLD. Liver biopsy is unrealistic to screen NAFLD in the general population for the prevalence of NAFLD. Previous studies also found that abdominal ultrasound is less expensive than other advanced imaging methods, and is currently the most widely used imaging tool in clinics and the most acceptable method for the first-line screening of steatosis. ${ }^{2}$ Furthermore, the participants could not provide their history of lipid-lowering therapy or antidiabetic drugs as 
they were old; therefore, information about therapy was missing from the data. Additionally, we could not evaluate the relationship between TyG index and different NAFLD severity. Also, we lacked other more accurate index which could reflect the abdominal obesity status. Even though we used WHtR to indicate abdominal obesity, we were unable to more accurately assess visceral fat index and the prevalence of NAFLD.

In conclusion, high baseline level of TyG index is significantly associated with a higher risk of NAFLD. In addition, TyG index plays a partial mediating role in the relationship between WHtR and NAFLD. Our results suggest that it has important clinical meaning for monitoring TyG index to prevent NAFLD.

\section{Author affiliations}

${ }^{1}$ Department of Nutrition and Food Hygiene, College of Public Health, Zhengzhou University, Zhengzhou, China

${ }^{2}$ Medical Record Room, Xinyang Central Hospital, Xinyang, China

${ }^{3}$ Jockey Club School of Public Health and Primary Care, The Chinese University of Hong Kong, New Territories, Hong Kong

${ }^{4}$ Department of Social Medicine, College of Public Health, Zhengzhou University, Zhengzhou, China

${ }^{5}$ Department of Pharmacy, Zhengzhou People's Hospital, Henan University of Traditional Chinese Medicine, Zhengzhou, China

${ }^{6}$ Health Commission of Xinzheng, Henan Province Health and Family Planning Commission, Zhengzhou, China

${ }^{7}$ Central for Disease Control of Xinzheng, Henan Province Center for Disease Control and Prevention, Zhengzhou, China

${ }^{8}$ Department of Epidemiology and Biostatistics, Zhengzhou University, Zhengzhou, China

${ }^{9}$ Department of Nutrition, The First Affiliated Hospital of Zhengzhou University, Zhengzhou University, Zhengzhou, China

Acknowledgements This research was funded by the National Natural Science Foundation of China (Grant No. 81673165), the National Key Research and Development Programme of China (Grant Nos 2017YFC1307705 and 2016YFC0106907). In addition, the authors are grateful to Junxing Fan for helping with the quality control of the data. The funders had no role in the study design, data collection and analysis, decision to publish or prepare the manuscript.

Contributors $\mathrm{CH}$ and $\mathrm{LQ}$ designed the study. CH, LS, BY, CX, SZ, WG and $\mathrm{HJ}$ participated in the data collection and analysis. $\mathrm{CH}$ and ANA drafted this manuscript, CH, LS and SS interpreted the data. SS and LQ reviewed and revised this manuscript. All authors approved the final manuscript.

Funding This research was funded by the National Natural Science Foundation of China (Grant No. 81673165), the National Key Research and Development Programme of China (Grant Nos 2017YFC1307705 and 2016YFC0106907).

Competing interests None declared.

Patient consent for publication Not required.

Ethics approval The study protocol was approved by Ethics Committee of Zhengzhou University in China (approval number: ZZURIB202004). Given the retrospective nature of the research, the requirement for informed consent was waived.

Provenance and peer review Not commissioned; externally peer-reviewed.

Data availability statement The data sets generated and/or analysed during the current study are available upon reasonable request.

Supplemental material This content has been supplied by the author(s). It has not been vetted by BMJ Publishing Group Limited (BMJ) and may not have been peer-reviewed. Any opinions or recommendations discussed are solely those of the author(s) and are not endorsed by BMJ. BMJ disclaims all liability and responsibility arising from any reliance placed on the content. Where the content includes any translated material, BMJ does not warrant the accuracy and reliability of the translations (including but not limited to local regulations, clinical guidelines, terminology, drug names and drug dosages), and is not responsible for any error and/or omissions arising from translation and adaptation or otherwise.

Open access This is an open access article distributed in accordance with the Creative Commons Attribution Non Commercial (CC BY-NC 4.0) license, which permits others to distribute, remix, adapt, build upon this work non-commercially, and license their derivative works on different terms, provided the original work is properly cited, appropriate credit is given, any changes made indicated, and the use is non-commercial. See: http://creativecommons.org/licenses/by-nc/4.0/.

\section{ORCID iDs}

Chen Xuejiao http://orcid.org/0000-0002-0669-3413

Shi Songhe http://orcid.org/0000-0002-2214-1440

\section{REFERENCES}

1 Finck BN. Targeting metabolism, insulin resistance, and diabetes to treat nonalcoholic steatohepatitis. Diabetes 2018;67:2485-93.

2 Yu Y, Cai J, She Z, et al. Insights into the epidemiology, pathogenesis, and therapeutics of nonalcoholic fatty liver diseases. Adv Sci 2019;6:1801585.

3 Castera L, Friedrich-Rust M, Loomba R. Noninvasive assessment of liver disease in patients with nonalcoholic fatty liver disease. Gastroenterology 2019;156:1264-81.

4 Anstee QM, Targher G, Day CP. Progression of NAFLD to diabetes mellitus, cardiovascular disease or cirrhosis. Nat Rev Gastroentero Hepatol 2013;10:330-44.

5 Schindhelm RK, Heine RJ, Diamant M. Prevalence of nonalcoholic fatty liver disease and its association with cardiovascular disease among type 2 diabetic patients. Diabetes Care 2007;30:e94.

6 Goldberg D, Ditah IC, Saeian K, et al. Changes in the prevalence of hepatitis $C$ virus infection, nonalcoholic steatohepatitis, and alcoholic liver disease among patients with cirrhosis or liver failure on the Waitlist for liver transplantation. Gastroenterology 2017;152:1090-9.

7 Watt MJ, Miotto PM, De Nardo W, et al. The liver as an endocrine Organ-Linking NAFLD and insulin resistance. Endocr Rev 2019;40:1367-93.

8 Baratta F, Pastori D, Polimeni L, et al. Adherence to Mediterranean diet and non-alcoholic fatty liver disease: effect on insulin resistance. Am J Gastroenterol 2017;112:1832-9.

9 Fedchuk L, Nascimbeni F, Pais R, et al. Performance and limitations of steatosis biomarkers in patients with nonalcoholic fatty liver disease. Aliment Pharmacol Ther 2014;40:1209-22.

10 Sanyal AJ. Past, present and future perspectives in nonalcoholic fatty liver disease. Nat Rev Gastroenterol Hepatol 2019;16:377-86.

11 Du T, Yuan G, Zhang M, et al. Clinical usefulness of lipid ratios, visceral adiposity indicators, and the triglycerides and glucose index as risk markers of insulin resistance. Cardiovasc Diabetol 2014;13:146.

12 Mohd Nor NS, Lee S, Bacha F, et al. Triglyceride glucose index as a surrogate measure of insulin sensitivity in obese adolescents with normoglycemia, prediabetes, and type 2 diabetes mellitus: comparison with the hyperinsulinemic-euglycemic clamp. Pediatr Diabetes 2016;17:458-65.

13 Kitae A, Hashimoto Y, Hamaguchi M, et al. The triglyceride and glucose index is a predictor of incident nonalcoholic fatty liver disease: a population-based cohort study. Can J Gastroenterol Hepatol 2019;2019:1-7.

14 Khoury M, Manlhiot C, McCrindle BW. Role of the waist/height ratio in the cardiometabolic risk assessment of children classified by body mass index. J Am Coll Cardiol 2013;62:742-51.

$15 \mathrm{Xi} \mathrm{B}, \mathrm{Mi}$ J, Zhao M, et al. Trends in abdominal obesity among U.S. children and adolescents. Pediatrics 2014;134:e334-9.

16 Huang X, Wang Q, Liu T, et al. Body fat indices as effective predictors of insulin resistance in obese/non-obese polycystic ovary syndrome women in the southwest of China. Endocrine 2019;65:81-5.

17 Motamed N, Rabiee B, Hemasi GR, et al. Body Roundness index and Waist-to-Height ratio are strongly associated with non-alcoholic fatty liver disease: a population-based study. Hepat Mon 2016;16:e39575.

18 Zheng R-D, Chen Z-R, Chen J-N, et al. Role of body mass index, Waist-to-Height and Waist-to-Hip ratio in prediction of nonalcoholic fatty liver disease. Gastroenterol Res Pract 2012;2012:1-6.

19 Zhang X, Wan Y, Zhang S, et al. Nonalcoholic fatty liver disease prevalence in urban school-aged children and adolescents from the Yangtze River delta region: a cross-sectional study. Asia Pac J Clin Nutr 2015;24:281-8.

20 Ramírez-Vélez R, Izquierdo M, Correa-Bautista JE, et al. Grip strength moderates the association between anthropometric and 
body composition indicators and liver fat in youth with an excess of adiposity. J Clin Med 2018;7. doi:10.3390/jcm7100347

21 Lin M-S, Lin T-H, Guo S-E, et al. Waist-to-height ratio is a useful index for nonalcoholic fatty liver disease in children and adolescents: a secondary data analysis. BMC Public Health 2017;17:851.

22 Procino F, Misciagna G, Veronese N, et al. Reducing NAFLDscreening time: a comparative study of eight diagnostic methods offering an alternative to ultrasound scans. Liver Int 2019;39:187-96.

23 Kojima S-I, Watanabe N, Numata M, et al. Increase in the prevalence of fatty liver in Japan over the past 12 years: analysis of clinical background. J Gastroenterol 2003;38:954-61.

24 Desquilbet L, Mariotti F. Dose-Response analyses using restricted cubic spline functions in public health research. Stat Med 2010;29:1037-57.

25 Jang E-H, Chang Y, Ryu S, et al. Cardiovascular health metrics in the development and regression of nonalcoholic fatty liver disease: a cohort study. J Clin Med 2019;8. doi:10.3390/jcm8050610

26 Petta S, Eslam M, Valenti L, et al. Metabolic syndrome and severity of fibrosis in nonalcoholic fatty liver disease: an age-dependent risk profiling study. Liver Int 2017;37:1389-96.

27 Simental-Mendía LE, Simental-Mendía E, Rodríguez-Hernández $\mathrm{H}$, et al. The product of triglycerides and glucose as biomarker for screening simple steatosis and NASH in asymptomatic women. Ann Hepatol 2016;15:715-20.

28 Zheng R, Du Z, Wang M, et al. A longitudinal epidemiological study on the triglyceride and glucose index and the incident nonalcoholic fatty liver disease. Lipids Health Dis 2018;17:262.

29 Zhang S, Du T, Zhang J, et al. The triglyceride and glucose index (TyG) is an effective biomarker to identify nonalcoholic fatty liver disease. Lipids Health Dis 2017;16:15.

30 Khan RS, Bril F, Cusi K, et al. Modulation of insulin resistance in nonalcoholic fatty liver disease. Hepatology 2019;70:711-24.

31 Sung K-C, Lee M-Y, Lee J-Y, et al. Natural course of fatty liver in 36,195 South Korean adults. Sci Rep 2019:9:9062.

32 Ballestri S, Nascimbeni F, Romagnoli D, et al. Type 2 Diabetes in Non-Alcoholic Fatty Liver Disease and Hepatitis C Virus Infection-Liver: The "Musketeer" in the Spotlight. Int J Mol Sci 2016;17:355.

33 Sookoian S, Pirola CJ. Systematic review with meta-analysis: risk factors for non-alcoholic fatty liver disease suggest a shared altered metabolic and cardiovascular profile between lean and obese patients. Aliment Pharmacol Ther 2017;46:85-95.

34 Wong VW-S, Chan W-K, Chitturi S, et al. Asia-Pacific Working Party on non-alcoholic fatty liver disease guidelines 2017-Part 1: definition, risk factors and assessment. J Gastroenterol Hepatol 2018;33:70-85.

35 Gastaldelli A. Insulin resistance and reduced metabolic flexibility: cause or consequence of NAFLD? Clin Sci 2017;131:2701-4.

36 Glass CK, Olefsky JM. Inflammation and lipid signaling in the etiology of insulin resistance. Cell Metab 2012;15:635-45.

37 Baeck C, Wehr A, Karlmark KR, et al. Pharmacological inhibition of the chemokine CCL2 (MCP-1) diminishes liver macrophage infiltration and steatohepatitis in chronic hepatic injury. Gut 2012;61:416-26.

38 Tang Z, Pham M, Hao Y, et al. Sex, age, and BMI modulate the association of physical examinations and blood biochemistry parameters and NAFLD: a retrospective study on 1994 cases observed at Shuguang Hospital, China. Biomed Res Int 2019;2019:1-7.

39 Liu M, Wang J, Zeng J, et al. Association of NAFLD with diabetes and the impact of BMI changes: a 5-year cohort study based on 18,507 elderly. J Clin Endocrinol Metab 2017;102:1309-16.

40 Shulman GI. Ectopic fat in insulin resistance, dyslipidemia, and cardiometabolic disease. N Engl J Med 2014;371:1131-41.

41 Caprio S, Perry R, Kursawe R. Adolescent obesity and insulin resistance: roles of ectopic fat accumulation and adipose inflammation. Gastroenterology 2017;152:1638-46.

42 Taksali SE, Caprio S, Dziura J, et al. High visceral and low abdominal subcutaneous fat stores in the obese adolescent: a determinant of an adverse metabolic phenotype. Diabetes 2008;57:367-71.

43 D'Adamo E, Cali AMG, Weiss R, et al. Central role of fatty liver in the pathogenesis of insulin resistance in obese adolescents. Diabetes Care 2010;33:1817-22.

44 Kursawe R, Dixit VD, Scherer PE, et al. A role of the inflammasome in the low storage capacity of the abdominal subcutaneous adipose tissue in obese adolescents. Diabetes 2016;65:610-8.

45 Stienstra R, van Diepen JA, Tack CJ, et al. Inflammasome is a central player in the induction of obesity and insulin resistance. Proc Natl Acad Sci U S A 2011;108:15324-9. 